\title{
Knot manifolds with isomorphic spines
}

\author{
by
}

\author{
Alberto Cavicchioli (Modena) and \\ Friedrich Hegenbarth (Milano)
}

\begin{abstract}
We study the relation between the concept of spine and the representation of orientable bordered 3-manifolds by Heegaard diagrams. As a consequence, we show that composing invertible non-amphicheiral knots yields examples of topologically different knot manifolds with isomorphic spines. These results are related to some questions listed in [9], [11] and recover the main theorem of [10] as a corollary. Finally, an application concerning knot manifolds of composite knots with $h$ prime factors completes the paper.
\end{abstract}

1. Introduction and notation. Throughout the paper we shall work in the piecewise linear (PL) category (see for example [13] and [22]). For standard definitions and results about knot theory we refer to [1], [8] and [12]. As general references about the topology of 3-manifolds see [7] and [17].

Let $K$ be an oriented tame (smooth or PL) knot in the right-hand oriented 3-sphere $\mathbb{S}^{3}$. Let $V=V(K)$ denote a tubular neighborhood of $K$ and $(\mu, \lambda)$ a standard meridian-longitude pair (preferred framing) of $V$ in $\mathbb{S}^{3}$. We always assume that the longitude $\lambda$ is oriented parallel to $K$ and the meridian $\mu$ is oriented so that its linking number with $K$ is +1 . The closed complement of $V$ in $\mathbb{S}^{3}$ is called the knot manifold of $K$, written $M=M(K)$. The fundamental group of $M$ is, by definition, the knot group of $K$, here denoted by $G=G(K)$. The triple $(G, \mu, \lambda)$, where the loops $\mu, \lambda$ are identified with their homotopy classes in $G$, is called the peripheral system of $K$. The inverse of $K$, denoted by $K^{-1}$, is the same knot with its orientation reversed. The mirror-image of $K$, denoted by

1991 Mathematics Subject Classification: Primary 57M25, 57Q40; Secondary 57N10, $57 \mathrm{M} 05$.

Key words and phrases: 3-manifold, spine, group presentation, Heegaard diagram, knot, knot group, knot manifold, peripheral system.

Work performed under the auspices of the G.N.S.A.G.A. of the C.N.R. and partially supported by the M.U.R.S.T. of Italy within the projects "Geometria reale e complessa" and "Topologia". 
$K^{*}$, is the image of $K$ under a reflection of $K$ in a plane. Two knots $K_{1}$ and $K_{2}$ are said to be equivalent if there exists a homeomorphism $f: \mathbb{S}^{3} \rightarrow \mathbb{S}^{3}$ such that $f\left(K_{1}\right)=K_{2}$. If $f$ preserves orientations, then we say that the knots are (ambient) isotopic and use the sign of equality.

We have the following basic results, proved in [4], [5], [6], [18] and [20].

TheOrem 1. Let $K_{i}, i=1,2$, be an oriented tame knot in the oriented 3-sphere, $\left(G_{i}, \mu_{i}, \lambda_{i}\right)$ the peripheral system and $M_{i}$ the knot manifold of $K_{i}$.

(1) $K_{1}$ is isotopic to $K_{2}$ if and only if there is an isomorphism of triples

$$
\left(G_{1}, \mu_{1}, \lambda_{1}\right) \simeq\left(G_{2}, \mu_{2}, \lambda_{2}\right) .
$$

(2) $K_{1}$ and $K_{2}$ are equivalent (resp. isotopic) if and only if $M_{1}$ is homeomorphic to $M_{2}$ (resp. by an orientation preserving homeomorphism).

By Theorem 1 knots are defined as purely algebraic objects by means of peripheral systems. For instance, given a knot $K=(G, \mu, \lambda)$, we have $K^{-1}=$ $\left(G, \mu^{-1}, \lambda^{-1}\right), K^{*}=\left(G, \mu, \lambda^{-1}\right)$ and $K^{-1 *}=\left(G, \mu^{-1}, \lambda\right)$. We observe that $K, K^{-1}, K^{*}$ and $K^{-1 *}$ are equivalent knots but they may not be isotopic. Thus, a knot $K$ is called invertible if $K=K^{-1}$, i.e. $(G, \mu, \lambda)$ is isomorphic to $\left(G, \mu^{-1}, \lambda^{-1}\right)$. A knot $K$ is said to be amphicheiral if $K=K^{*}$, i.e. $(G, \mu, \lambda)$ is isomorphic to $\left(G, \mu, \lambda^{-1}\right)$.

The notion of composition (also named product) of knots is well defined on the oriented isotopy type of a knot. Thus the (oriented) connected sum of two knots $K_{i}=\left(G_{i}, \mu_{i}, \lambda_{i}\right), i=1,2$, is defined to be

$$
K_{1} \# K_{2}=\left(G_{1} \underset{\mu_{1}=\mu_{2}}{*} G_{2}, \mu_{1}, \lambda_{1} \lambda_{2}\right)
$$

where the symbol $* \mu_{1}=\mu_{2}$ denotes the amalgamated free product. A knot is called composite if it is a composition of two non-trivial knots; a non-trivial knot which is not composite is said to be prime.

The following results are well known (see [1], [5], [14], [15] and [19]).

Theorem 2. (1) (The unique prime decomposition theorem) Any nontrivial knot in the 3-sphere is a finite product of prime knots and the isotopy types of the factors are uniquely determined up to permutation.

(2) (The Whitten rigidity theorem) Prime knots in the 3-sphere with isomorphic groups have homeomorphic knot manifolds, i.e. they are equivalent.

(3) If two knots have isomorphic groups, then either both knots are prime or both are composite.

Now let $M$ be a compact connected (triangulated) 3-manifold with nonvoid boundary $\partial M$. A compact 2-polyhedron $P$ is called a spine of $M$ if there exists an embedding $f: P \rightarrow \operatorname{int} M$ such that $M \backslash f(P)$ is homeomorphic to $\partial M \times[0,1[$. It is known that composing certain torus knots, via 
connected sums, yields examples of topologically different knot manifolds which have a common spine (see [2], [10], [11]). These facts suggest studying the following problem, which is related to some questions listed in [9] and [11]. Consider two tame knots $K_{1}, K_{2}$ in the 3 -sphere $\mathbb{S}^{3}$ and let $M_{1}, M_{2}$ be the knot manifolds of $K_{1}, K_{2}$ respectively. Then we study the conditions on which $M_{1}, M_{2}$ possess a common spine but are not homeomorphic. Obviously, the considered knots must be composite by the Whitten rigidity theorem. Our main result states that any Wirtinger presentation of a knot group corresponds, in a natural way, to a spine of the knot manifold. This implies the main theorem of [10] as a corollary. Then we give examples of non-homeomorphic knot manifolds with isomorphic spines, by composing prime knots which are not necessarily toroidal as in [10], [11]. Finally, an application for composite knots with $h(h \geq 1)$ prime factors completes the paper.

2. Spines of bordered manifolds. Let $\eta$ be a group presentation with $n$ generators $x_{1}, \ldots, x_{n}$ and $m$ relators $r_{1}, \ldots, r_{m}, n \geq m$. Let $P_{\eta}$ denote the canonical 2-polyhedron associated with $\eta$. Then $P_{\eta}$ is a 2-dimensional cell complex with one vertex $v$ and $n$ oriented 1-cells (resp. $m$ 2-cells) corresponding to generators (resp. relators) of $\eta$. We shall always label each 1-cell of $P_{\eta}$ by the corresponding generator $x_{i}$ of $\eta$. Let $c_{1}, \ldots, c_{m}$ be disjoint 2-cells. Then there is an attaching map $\varphi_{j}: \partial c_{j} \rightarrow \bigvee_{i} x_{i}$ which sends $\partial c_{j}$ to the word $r_{j}$ of $\eta$, for each $j=1, \ldots, m$. Here the symbol $\bigvee_{i} x_{i}$ represents the wedge of the oriented loops $x_{i}$.

Thus we have

$$
P_{\eta}=\left(\bigvee_{i} x_{i}\right) \cup_{\varphi}\left(\bigcup_{j} c_{j}\right)
$$

where $\varphi$ is the union of the maps $\varphi_{j}$.

Let now $M$ be a compact connected orientable (PL) 3-manifold with non-void boundary $\partial M$. In this section, we give a simple geometric criterion to recognize when the polyhedron $P_{\eta}$ is a spine of $M$. For this, we briefly recall some definitions relating to the representation of bordered 3-manifolds by Heegaard diagrams (see for example [17]). A Heegaard diagram of genus $n$ is a pair $(H, \gamma)$, where $H$ is an (orientable) cube with $n$ handles and $\gamma$ is a set of disjoint simple closed curves $\gamma_{j}, j=1, \ldots, m$, on $\partial H, n \geq m$. The diagram determines a unique (up to homeomorphism) compact (orientable) 3 -manifold $M$ with non-void boundary $\partial M$. Indeed, for each $j=1, \ldots, m$, let $B_{j}$ be a 2-cell and $N_{j}$ an annular neighborhood of $\gamma_{j}$ in $\partial H$. Then $M$ is obtained from $H$ by glueing the plates $B_{j} \times I(I=[0,1])$ to $N_{j}$ via homeomorphisms $\psi_{j}: \partial B_{j} \times I \rightarrow N_{j}$. 
Thus we have

$$
M=H \cup_{\psi} \bigcup_{j}\left(B_{j} \times I\right)
$$

where $\psi$ is the union of the attaching maps $\psi_{j}$. It is well known that any compact orientable bordered 3-manifold can be constructed in this way (see for example [17]).

Now we prove the following result.

THEOREM 3. Let $\eta$ be a group presentation with $n$ generators and $m$ relators $(n \geq m)$ and let $P_{\eta}$ be the canonical 2-polyhedron associated with $\eta$. Then $P_{\eta}$ is a spine of a compact orientable 3-manifold $M$ with nonvoid boundary $\partial M$ if and only if $\eta$ arises from a Heegaard diagram of $M$. Furthermore, if $M$ has $h$ boundary components, then the genus of $\partial M$ equals $n-m+h-1$.

Proof. Necessity is a consequence of Theorem 7 of [2]. We sketch the proof of this result to make the reading clear. Let $U, V_{i}$ be regular neighborhoods of $v, x_{i}$ in $P_{\eta}$ respectively and denote the points of $x_{i} \cap \partial U$ by $e_{i}$ and $\bar{e}_{i}$. Let $e_{i}^{k}, \bar{e}_{i}^{k}$ be the points of $\partial V_{i}$ which lie in regular neighborhoods of $e_{i}, \bar{e}_{i}$ in $\partial U$ respectively, $k=1, \ldots, \alpha(i), i=1, \ldots, n$. Further, we can suppose that $e_{i}^{k}$ and $\bar{e}_{i}^{k}$ are joined by an arc in $\partial V_{i} \backslash U$. Then we set $E_{i}=\left\{e_{i}^{k}: k=1, \ldots, \alpha(i)\right\}, \bar{E}_{i}=\left\{\bar{e}_{i}^{k}: k=1, \ldots, \alpha(i)\right\}$ and $E=\bigcup_{i=1}^{n}\left(E_{i} \cup \bar{E}_{i}\right)$. A simple curve near each $\partial c_{j}$ intersects $\partial U$ in a set of simple $\operatorname{arcs} \beta_{r}$ with endpoints in $E$. Interchanging the endpoints of these arcs defines an involution $A=A(\eta)$ of $E$. Let $B=B(\eta)$ be the involutory permutation of $E$ defined by $B\left(e_{i}^{k}\right)=\bar{e}_{i}^{k}$. An arbitrary numbering of the elements of $E$ around each vertex $e_{i}$ (resp. $\bar{e}_{i}$ ) determines a permutation $C=C(\eta)$ of $E$, whose orbit sets are $E_{i}, \bar{E}_{i}, i=1, \ldots, n$. Theorem 7 of [2] states that $P_{\eta}$ is a spine of a compact orientable 3-manifold $M$ with $h$ boundary components if and only if the following conditions hold:

(1) the number of orbits of the permutation group generated by $A C$ and $B C$ equals $h$;

(2) $|A|-|C|+2=|A C|,|\theta|$ being the number of cycles of a permutation $\theta: E \rightarrow E$.

As a consequence, the genus of $\partial M$ is proved to be $n-m+h-1$. Furthermore, $M$ is prime if and only if the permutations $A$ and $C$ generate a transitive group. As remarked in [2], the permutations $A$ and $C$ yields a Heegaard diagram $(H, \gamma)$ of $M$ from which one can read off the presentation $\eta$ of the fundamental group $\Pi_{1}(M)$. Indeed, the unbarred cycles of $C$ represent the holes of $H$ and the set of $\operatorname{arcs} \beta_{r}$, joining $A$-correspondent points of $E$, fit the curves $\gamma_{j}$ of the diagram (for details see [2]). This completes the proof of necessity. 
Conversely, suppose that $\eta=\left\langle x_{1}, \ldots, x_{n}: r_{1}, \ldots, r_{m}\right\rangle$ arises from a Heegaard diagram $(H, \gamma)$ of a bordered orientable 3 -manifold $M$. Then $x_{1}$, $\ldots, x_{n}$ are the oriented loops of a wedge on which $H$ collapses, i.e. they generate the free group $\Pi_{1}(H) \simeq *_{n} \mathbb{Z}$. The relator $r_{j}$ is precisely the element of $\Pi_{1}(H)$ corresponding to the curve $\gamma_{j}=\psi_{j}\left(\partial B_{j} \times \frac{1}{2}\right)$ for each $j=1, \ldots, m$. Because each plate $B_{j} \times I$ collapses onto $B_{j} \times \frac{1}{2}$, it follows that $M$ collapses onto the subpolyhedron

$$
Q=H \cup_{\psi} \bigcup_{j}\left(B_{j} \times \frac{1}{2}\right)
$$

where $\psi$ also denotes the union of the restrictions $\psi_{j}: \partial B_{j} \times \frac{1}{2} \rightarrow \partial H$. Because $H$ collapses onto the wedge $\bigvee_{i} x_{i}$ by moves outside the open attached 2-cells, the polyhedron $Q$ (and hence $M$ ) collapses onto $P_{\eta}$. This proves that $P_{\eta}$ is a spine of $M$. In this process, we may deform a triangulation of $Q$ over itself so as to contract a maximal tree in its 1-skeleton to a single vertex, $v$ say. This deformation can be extended to one of $Q$ over itself with the resulting space being homeomorphic to $Q$. Thus the proof is complete.

EXAMPLE. In Fig. 1 we draw a regular projection of the 2-bridge knot $\mathbf{b}(7,3)=5_{2}$ (see [1], Appendix D).

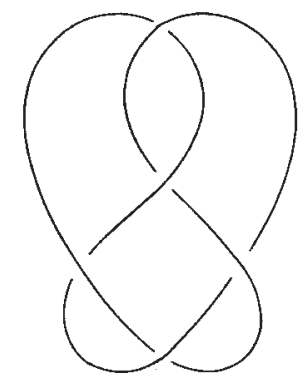

Fig. 1. The 2-bridge knot $\mathbf{b}(7,3)=5_{2}$

It is well known that $5_{2}$ is an invertible non-amphicheiral knot with signature $\sigma\left(5_{2}\right)=2$. Furthermore, the knot $5_{2}$ is not toroidal since its Alexander polynomial is $2 t^{2}-3 t+2$. In Fig. 2 we show a Heegaard diagram of the knot manifold $M$ of $5_{2}$, constructed by the algorithm given in [2]. This diagram induces the presentation

$$
\eta=\left\langle x, y: x y x y^{-1} x^{-1} y x y^{-1} x^{-1} y^{-1} x y x^{-1} y^{-1}\right\rangle
$$

of the fundamental group $\Pi_{1}(M)$. Thus Theorem 3 implies that the canonical 2-polyhedron $P_{\eta}$ is a spine of the knot manifold $M$. 


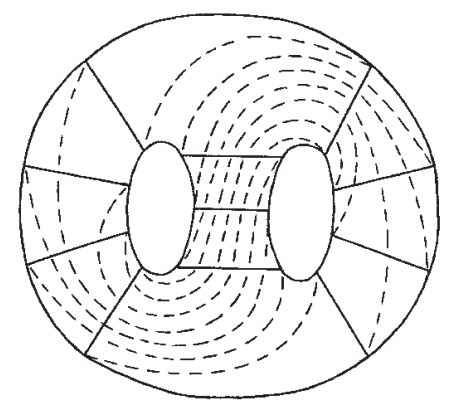

Fig. 2. A Heegaard diagram (full outside) of the knot manifold of the knot $5_{2}$

3. Knot manifolds. Now we prove the results announced in Section 1.

TheOREM 4. Let $K$ be an oriented tame knot in the oriented 3-sphere. Suppose that $\eta$ is a Wirtinger presentation of the knot group of $K$. Then the canonical 2-polyhedron $P_{\eta}$ is a spine of the knot manifold of $K$.

Proof. By Theorem 3 we have to show that any Wirtinger presentation $\eta$ of the knot group arises from a Heegaard diagram of the knot manifold. For convenience, we first assume that the knot $K$ is embedded in the euclidean 3 -space $\mathbb{R}^{3}$, where $\mathbb{S}^{3}=\mathbb{R}^{3} \cup\{\infty\}$. Following [12], we recall the procedure for writing a Wirtinger presentation $\eta$ of the knot group of $K$. Let $\alpha_{1}, \ldots$, $\alpha_{n}$ denote the oriented arcs of a regular projection of the knot, also named $K$, in the plane $z=0$. As usual, each $\alpha_{i}$ is assumed connected to $\alpha_{i-1}$ and $\alpha_{i+1}$ (indices $\bmod n$ ) by undercrossing $\operatorname{arcs} \beta_{i}$ and $\beta_{i+1}$. We also assume that the orientations of the $\operatorname{arcs} \alpha_{i}$ are compatible with the order of their subscripts and that $\beta_{i}$ lies in the plane $z=-\varepsilon$ for some $\varepsilon>0$.

If $V$ is a tubular neighborhood of $K$ in $\mathbb{R}^{3}$, then we dissect the knot complement $\mathbb{R}^{3} \backslash$ int $V$ into pieces $X, Y_{1}, \ldots, Y_{n}, Z$ as shown in [12]. Let $\Sigma$ denote the upper semispace of $\mathbb{R}^{3}$ having the plane $z=-\varepsilon$ as origin. Then $X$ is defined to be the closure of the complement of $V$ in $\Sigma$. Let $x_{i}$ be an oriented loop in $X$ passing under $\alpha_{i}$ in a right-left direction and with base point $v \equiv(0,0,1)$. Obviously $X$ collapses onto the wedge $\bigvee_{i} x_{i}$, hence the compact orientable 3-manifold $\widetilde{X}=X \cup\{\infty\}$ is a cube with $n$ handles. At each undercrossing of $K$, we consider a solid rectangular box $R_{i}$, whose top lies on the plane $z=-\varepsilon$ and surrounds $\beta_{i}$.

Let $Y_{i}$ denote the 3-cell obtained from $R_{i}$ by removing a small regular neighborhood (in $R_{i}$ ) of $\beta_{i}$. The cells $Y_{i}$ may be taken to be disjoint from one another. Let $\gamma_{i} \subset Y_{i} \cap X$ be an oriented loop whose homotopy class generates the free group $\Pi_{1}\left(Y_{i} \cap X\right) \simeq \mathbb{Z}$. Now the cell $Y_{i}$ is homeomorphic to a plate $B_{i} \times I$, whose rim $\partial B_{i} \times \frac{1}{2}$ maps onto the curve $\gamma_{i}$. Moreover, the intersection $Y_{i} \cap X$ is an annular neighborhood of $\gamma_{i}$ in $\partial Y_{i}$. 
Finally, let $Z$ denote the closure of everything below the union $X \cup$ $\bigcup_{i=1}^{n} Y_{i}$ and set $\widetilde{Z}=Z \cup\{\infty\}$. Now the space $\widetilde{X} \cup \widetilde{Z} \cup \bigcup_{i=1}^{n} Y_{i}$ is precisely the knot manifold $M$ of $K \subset \mathbb{S}^{3}$. Because $\widetilde{Z} \cup Y_{n}$ is a closed 3-cell which intersects $\partial M$ in a closed 2-cell, $M$ is (PL) homeomorphic to $\widetilde{X} \cup \bigcup_{j=1}^{n-1} Y_{j}$. Thus $M$ is obtained from the cube with handles $\widetilde{X}$ by glueing the plates $Y_{j}$ along annular neighborhoods of their rims $\gamma_{j}$, for $j=1, \ldots, n-1$. When $\gamma_{j}$ is included in $\widetilde{X}$, it becomes the Wirtinger word

$$
r_{j}=x_{k}^{\xi} x_{j+1} x_{k}^{-\xi} x_{j}^{-1},
$$

where $\xi= \pm 1$ and $\alpha_{k}$ is the arc passing over the gap from $\alpha_{j}$ to $\alpha_{j+1}(k=j$ or $j+1$ is possible). If $\gamma$ is the set of all the curves $\gamma_{j}, j=1, \ldots, n-1$, then the pair $(\widetilde{X}, \gamma)$ is a Heegaard diagram (of genus $n$ ) of $M$. Obviously, this diagram induces the Wirtinger presentation $\eta$ of the knot group $\Pi_{1}(M)$. Thus the proof is complete.

The following result implies the main theorem of [10] as a corollary.

Proposition 5. Let $K_{1} \subset \mathbb{S}^{3}$ be an invertible non-amphicheiral knot and let $K_{2} \subset \mathbb{S}^{3}$ be an arbitrary knot. Then the knot manifolds of $K_{1} \# K_{2}$ and $K_{1}^{*} \# K_{2}$ admit a common spine. If $K_{2}$ is non-amphicheiral, then the knot manifolds are not homeomorphic.

Proof. By Theorem 4, it suffices to prove that the composite knots $K_{1} \# K_{2}$ and $K_{1}^{*} \# K_{2}$ have the same Wirtinger presented group. If $K_{i}=$ $\left(G_{i}, \mu_{i}, \lambda_{i}\right), i=1,2$, then we have

$$
K_{1} \# K_{2}=\left(G_{1} \underset{\mu_{1}=\mu_{2}}{*} G_{2}, \mu_{1}, \lambda_{1} \lambda_{2}\right), \quad K_{1}^{*} \# K_{2}=\left(G_{1} \underset{\mu_{1}^{-1}=\mu_{2}}{*} G_{2}, \mu_{2}, \lambda_{1} \lambda_{2}\right) .
$$

Because $K_{1}$ is invertible, the knot group $G_{1}$ admits an automorphism $\varphi$ such that $\varphi\left(\mu_{1}\right)=\mu_{1}^{-1}$ and $\varphi\left(\lambda_{1}\right)=\lambda_{1}^{-1}$. Thus the groups of the composite knots are isomorphic. We observe that the peripheral systems may not be isomorphic. Now let

$$
\eta_{1}=\left\langle x_{1}, \ldots, x_{n}: r_{1}, \ldots, r_{n-1}\right\rangle, \quad \eta_{2}=\left\langle y_{1}, \ldots, y_{m}: s_{1}, \ldots, s_{m-1}\right\rangle
$$

be Wirtinger presentations of $G_{1}$ and $G_{2}$ respectively, where $\mu_{1}=x_{n}$ and $\mu_{2}=y_{m}$. Then the amalgamated free product

$$
G_{1} \underset{\mu_{1}=\mu_{2}}{*} G_{2} \simeq G_{1} \underset{\mu_{1}^{-1}=\mu_{2}}{*} G_{2}
$$

is presented by

$$
\left\langle x_{1}, \ldots, x_{n}, y_{1}, \ldots, y_{m-1}: r_{1}, \ldots, r_{n-1}, s_{1}^{\prime}, \ldots, s_{m-1}^{\prime}\right\rangle,
$$

where $s_{j}^{\prime}$ is the word obtained from $s_{j}$ by replacing the generator $y_{m}$ (if it occurs) with $x_{n}$. 
Suppose now that $K_{2}$ is non-amphicheiral. Because $K_{1}$ is non-amphicheiral, the unique prime decomposition theorem implies that $K_{1} \# K_{2}$ and $K_{1}^{*} \# K_{2}$ are not isotopic. Hence there is no orientation preserving homeomorphism between the knot manifolds of these knots. On the other hand, any orientation reversing homeomorphism induces an orientation preserving one between the knot manifolds of $K_{1} \# K_{2}$ and $\left(K_{1}^{*} \# K_{2}\right)^{*}=K_{1} \# K_{2}^{*}$. This contradicts the fact that $K_{1} \# K_{2}$ and $K_{1} \# K_{2}^{*}$ are not isotopic whenever $K_{2}$ is non-amphicheiral. Thus we have proved that the knot manifolds of the statement are topologically different.

As a direct consequence of Proposition 5, we have the following result.

COROLlaRY 6. Let $K \subset \mathbb{S}^{3}$ be an invertible non-amphicheiral knot in the oriented 3 -sphere. Then the knot manifolds of $K \# K$ and $K^{*} \# K$ are not homeomorphic but they do possess a common spine.

EXAMPle. Corollary 6 applies for $K=5_{2}$. In Fig. 3 we show regular projections of the composite knots $5_{2} \# 5_{2}$ and $5_{2}^{*} \# 5_{2}$.
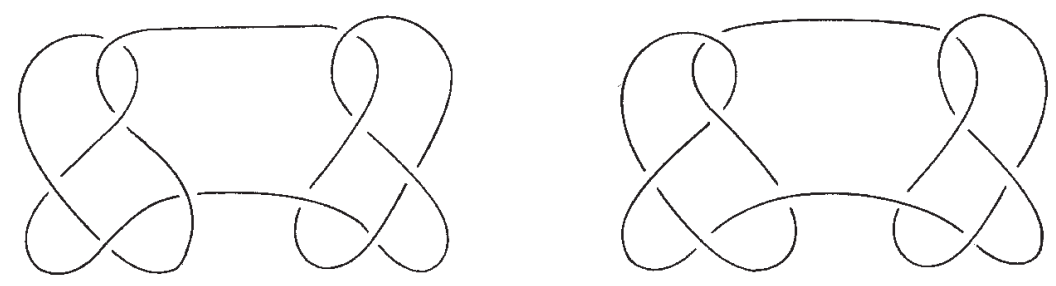

Fig. 3 . The composite knots $5_{2} \# 5_{2}$ and $5_{2}^{*} \# 5_{2}$

Figures 4 and 5 show Heegaard diagrams of the knot manifolds of $5_{2} \# 5_{2}$ and $5_{2}^{*} \# 5_{2}$ respectively. Both the diagrams induce the presentation

$$
\begin{array}{r}
\eta=\left\langle x, y, z: x y x y^{-1} x^{-1} y x y^{-1} x^{-1} y^{-1} x y x^{-1} y^{-1},\right. \\
\left.y z y z^{-1} y^{-1} z y z^{-1} y^{-1} z^{-1} y z y^{-1} z^{-1}\right\rangle
\end{array}
$$

of the knot group. We observe that a Wirtinger presentation is not very economical with respect to the genus of the Heegaard diagrams. Thus to produce the examples we have used extended Nielsen transformations on group presentations. These moves yield simplified presentations which also arise from Heegaard diagrams of the same manifold (for details see [3], [16] and $[21])$.

Proposition 7. Let $K_{1}$ and $K_{2}$ be oriented tame knots in the oriented 3 -sphere. Then the knot manifolds of $K_{1} \# K_{2}$ and $K_{1} \# K_{2}^{-1 *}$ (resp. $K_{1} \# K_{2}^{*}$ and $K_{1} \# K_{2}^{-1}$ ) admit a common spine. If the signature $\sigma\left(K_{i}\right)$ of $K_{i}$ is not null, $i=1,2$, then the knot manifolds are not homeomorphic. 


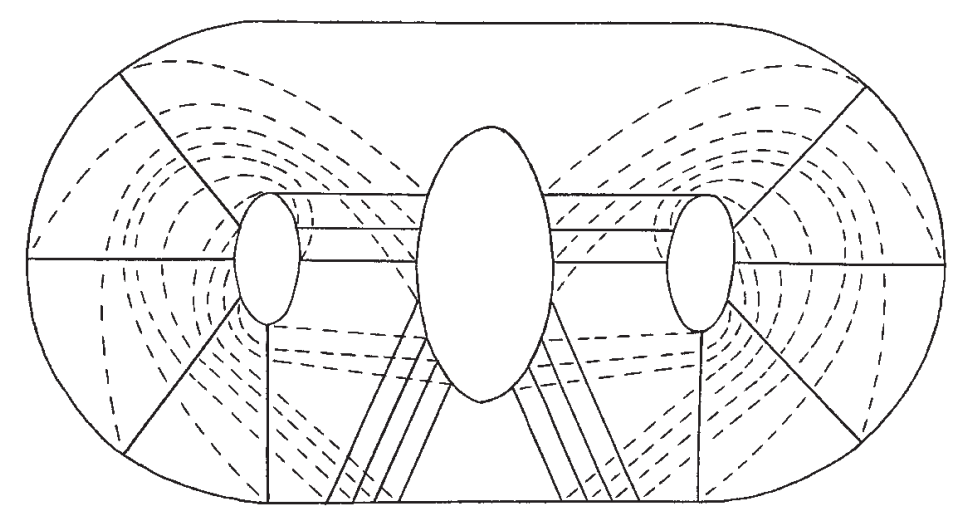

Fig. 4. A Heegaard diagram of the knot manifold of the knot $5_{2} \# 5_{2}$

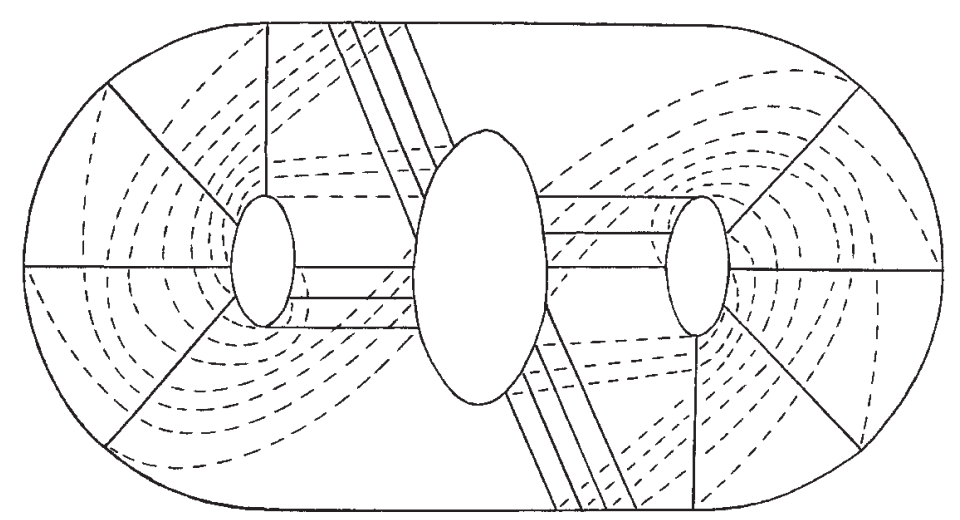

Fig. 5. A Heegaard diagram of the knot manifold of the knot $5_{2}^{*} \# 5_{2}$

Proof. The knot groups of the first (resp. second) pair of knots admit a common Wirtinger presentation. Indeed, we have the following relations:

$$
\begin{aligned}
K_{1} \# K_{2} & =\left(G_{1} \underset{\mu_{1}=\mu_{2}}{\stackrel{*}{=}} G_{2}, \mu_{1}, \lambda_{1} \lambda_{2}\right), \\
K_{1} \# K_{2}^{-1 *} & =\left(G_{1} \underset{\mu_{1}}{\stackrel{*}{*} \mu_{2}} G_{2}, \mu_{1}, \lambda_{1} \lambda_{2}^{-1}\right), \\
K_{1} \# K_{2}^{*} & =\left(G_{1} \underset{\mu_{1}=\mu_{2}^{-1}}{*} G_{2}, \mu_{1}, \lambda_{1} \lambda_{2}\right), \\
K_{1} \# K_{2}^{-1} & =\left(G_{1}^{\underset{\mu_{1}=\mu_{2}^{-1}}{*}} G_{2}, \mu_{1}, \lambda_{1} \lambda_{2}^{-1}\right) .
\end{aligned}
$$

If $\sigma\left(K_{2}\right) \neq 0$, then $K_{1} \# K_{2}$ and $K_{1} \# K_{2}^{-1 *}\left(\right.$ resp. $K_{1} \# K_{2}^{*}$ and $K_{1} \# K_{2}^{-1}$ ) are not isotopic since they have different signatures (use the additivity of $\sigma$ and the properties $\left.\sigma(K)=\sigma\left(K^{-1}\right), \sigma\left(K^{*}\right)=-\sigma(K)\right)$.

If $\sigma\left(K_{1}\right) \neq 0$, then $K_{1} \# K_{2}$ and $\left(K_{1} \# K_{2}^{-1 *}\right)^{*}=K_{1}^{*} \# K_{2}^{-1}$ (resp. $K_{1} \# K_{2}^{*}$ and $\left.\left(K_{1} \# K_{2}^{-1}\right)^{*}=K_{1}^{*} \# K_{2}^{-1 *}\right)$ are not isotopic by the same reasons as 
above. Thus we have proved that the composite knots, in the statement, are not equivalent, i.e. their knot manifolds are topologically different. This completes the proof.

Now we close the paper with a result about spines of knot manifolds of composite knots with $h$ prime factors. It is a direct consequence of our Proposition 5 and of theorems proved in [14], [15].

Proposition 8. Let $K$ be an oriented composite knot with $h(h \geq 1)$ prime factors, in the oriented 3-sphere. Let $\eta$ denote a Wirtinger presentation of the knot group of $K$. Then the canonical 2-polyhedron $P_{\eta}$ is a spine of at most $2^{h-1}$ topologically different knot manifolds. This bound is actually attained by composing invertible non-amphicheiral distinct knots.

\section{References}

[1] G. Burde and H. Zieschang, Knots, Walter de Gruyter, Berlin, 1985.

[2] A. Cavicchioli, Imbeddings of polyhedra in 3-manifolds, Ann. Mat. Pura Appl. 162 (1992), 157-177.

[3] R. Craggs, Free Heegaard diagrams and extended Nielsen transformations, I, Michigan Math. J. 26 (1979), 161-186; II, Illinois J. Math. 23 (1979), 101-127.

[4] M. Culler, C. Mc A. Gordon, J. Luecke and P. Shalen, Dehn surgery on knots, Bull. Amer. Math. Soc. 13 (1985), 43-45; Ann. of Math. 125 (1987), 237-300.

[5] C. D. Feustel and W. Whitten, Groups and complement of knots, Canad. J. Math. 30 (1978), 1284-1295.

[6] C. Mc A. Gordon and J. Luecke, Knots are determined by their complements, Bull. Amer. Math. Soc. 20 (1989), 83-87; J. Amer. Math. Soc. 2 (1989), 371-415.

[7] J. Hempel, 3-manifolds, Princeton Univ. Press, Princeton, N.J., 1976.

[8] L. H. Kauffman, On knots, Ann. of Math. Stud. 115, Princeton Univ. Press, Princeton, N.J., 1987.

[9] R. Kirby, Problems in low dimensional manifold theory, in: Proc. Sympos. Pure Math. 32, Amer. Math. Soc., Providence, R.I., 1978, 273-312.

[10] W. J. R. Mitchell, J. Przytycki and D. Repovš, On spines of knot spaces, Bull. Polish Acad. Sci. 37 (1989), 563-565.

[11] D. Repov̌s, Regular neighbourhoods of homotopically PL embedded compacta in 3-manifolds, Suppl. Rend. Circ. Mat. Palermo 18 (1988), 415-422.

[12] D. Rolfsen, Knots and Links, Math. Lecture Ser. 7, Publish or Perish, Berkeley, 1976.

[13] T. B. Rushing, Topological Embeddings, Academic Press, New York, 1973.

[14] J. Simon, On the problems of determining knots by their complements and knot complements by their groups, Proc. Amer. Math. Soc. 57 (1976), 140-142.

[15] -, How many knots have the same group?, ibid. 80 (1980), 162-166.

[16] J. Singer, Three-dimensional manifolds and their Heegaard diagrams, Trans. Amer. Math. Soc. 35 (1933), 88-111.

[17] J. Stillwell, Classical Topology and Combinatorial Group Theory, Springer, New York, 1980.

[18] F. Waldhausen, On irreducible 3-manifolds which are sufficiently large, Ann. of Math. 87 (1968), 56-88. 
[19] W. Whitten, Rigidity among prime-knot complements, Bull. Amer. Math. Soc. 14 (1986), 299-300.

[20] - Knot complements and groups, Topology 26 (1987), 41-44.

[21] P. Wright, Group presentations and formal deformations, Trans. Amer. Math. Soc. 208 (1975), 161-169.

[22] E. C. Zeeman, Seminar on Combinatorial Topology, mimeographed notes, Inst. des Hautes Études Sci., Paris, 1963.

DIPARTIMENTO DI MATEMATICA

UNIVERSITÀ DI MODENA

VIA CAMPI 213/B

41100 MODENA, ITALY
DIPARTIMENTO DI MATEMATICA UNIVERSITÀ DI MILANO VIA SALDINI 50 20133 MILANO, ITALY

Received 17 June 1993 